\title{
INFRARED CEPHALIC-VEIN TO ASSIST BLOOD EXTRACTION TASKS: AUTOMATIC PROJECTION AND RECOGNITION
}

\author{
S. Lagüela ${ }^{\mathrm{a}, \mathrm{b}}, \mathrm{M}$ Gesto $^{\mathrm{b}}, \mathrm{B}$. Riveiro ${ }^{\mathrm{a} *}, \mathrm{D}$. González-Aguilera ${ }^{\mathrm{b}}$ \\ a Applied Geotechnologies Research Group, University of Vigo, 36310 Vigo, Spain - (susiminas, belenriveiro)@uvigo.es \\ ${ }^{b}$ Dept. of Cartographic and Terrain Engineering, University of Salamanca, 05001 Ávila, Spain - (sulaguela, mgesto,
} daguilera)@usal.es

Commission II, WG II/10

KEY WORDS: infrared thermography; projector; pattern recognition; cephalic vein; thermal map.

\begin{abstract}
:
Thermal infrared band is not commonly used in photogrammetric and computer vision algorithms, mainly due to the low spatial resolution of this type of imagery. However, this band captures sub-superficial information, increasing the capabilities of visible bands regarding applications. This fact is especially important in biomedicine and biometrics, allowing the geometric characterization of interior organs and pathologies with photogrammetric principles, as well as the automatic identification and labelling using computer vision algorithms.

This paper presents advances of close-range photogrammetry and computer vision applied to thermal infrared imagery, with the final application of Augmented Reality in order to widen its application in the biomedical field. In this case, the thermal infrared image of the arm is acquired and simultaneously projected on the arm, together with the identification label of the cephalic-vein. This way, blood analysts are assisted in finding the vein for blood extraction, especially in those cases where the identification by the human eye is a complex task. Vein recognition is performed based on the Gaussian temperature distribution in the area of the vein, while the calibration between projector and thermographic camera is developed through feature extraction and pattern recognition. The method is validated through its application to a set of volunteers, with different ages and genres, in such way that different conditions of body temperature and vein depth are covered for the applicability and reproducibility of the method.
\end{abstract}

\section{INTRODUCTION}

\subsection{Thermal infrared thermography}

Infrared thermography is the science to measure the radiation emitted by bodies in the thermal infrared band of the spectrum. Bodies emit radiation in this band as a function of their temperature, with proportional relation (higher temperatures imply more intensity of thermal radiation).

The applications of infrared thermography are diverse and cover all fields, from architecture to agriculture, including industrial, mechanical and electrical maintenance (Lagüela et al, 2016). Regarding health and sanitary fields, the first applications of infrared thermography was sports medicine (Hildebrandt et al, 2010). The basis of the application was that inflammation provokes the accumulation of blood in the area, with a consequent increase in temperature. Veterinary science was the second application of infrared thermography regarding health, especially applied to detect injuries in horses and other mammals (McCafferty, 2007). The application was justified by the high cost of these animals and the expensive consequences of their physical disability.

Advances in the technique have led to the most recent application, which is the detection of cancerogenic tumours. These imply an irregular temperature distribution in the area, which can be detected in a thermographic image provided they present a subsuperficial depth, as in oral and breast cancer. Studies have used different features, from texture to temperature dissimilarities, for cancer detection (Chakraborty et al, 2016; Araujo et al, 2014).

The differences in temperature caused by vascularization anomalies have also been analysed for the thermographic detection of problems related to thrombosis and the study of the effect of the injection of substances in the veins (Urakov et al, 2014). Regarding advances in computer science, dorsal hand vein detection has been exploited for biometrics and bioinformatics purposes (Alpar \& Krejcar, 2017).

Computer vision has played an important role in these advances, through the application of its algorithms to thermal infrared images to improve the detection of thermal anomalies and their source pathology (Dayakshini et al, 2016).

\subsection{Augmented reality}

Augmented Reality, AR, consists on the addition of semantics or complementary information to the real view of the environment, in such way that the viewer has information beyond reality. Although the first applications of augmented reality were designed for desktop-computer users allowing multiple views, advances in technology led to the generation of portable devices for personalized visualization of the information. (Kato \& Billinghurst, 1999) designed a device for collaborative viewing, where desktop-computer users are provided the information viewed by a user with a head-mounted display.

The applications of AR in medicine are wide, mainly based on providing visual information of the interior of the body to the doctor or surgeon (Thomas, 2016). In addition, AR has proved its utility for medical training, allowing trainees to practice operations and related tasks in virtual bodies, thus minimizing the risk of failure (Barsom et al, 2016). AR-based training can also optimize and improve training activities, by providing tips according to the level and learning curve of the trainee.

The combination of infrared thermography with AR has mainly being applied to buildings and manufacturing, allowing the performance of efficient inspections regarding energy efficiency and electrical installations (Borrmann et al, 2016).

This paper presents an application of augmented reality in the biomedical field, combining infrared thermography data and 
computer vision algorithms. The objective of the study is the generation of a thermographic assistance system for blood extraction tasks, based on the capability of thermal infrared to detect blood vessels due to their thermal contrast with the other body tissues.

\section{MATERIALS AND METHODS}

\subsection{Thermographic assistance system}

The system designed in for assistance in blood extraction tasks consists on a thermographic camera and a projector, both controlled via PC. The camera and the projector are fixed and installed on an aluminium structure, made of square profiles with rails, as shown in Figure 1. The rails are used to fix the camera and the projector to the structure with framing squares and screws.

The thermographic camera used for the study is a Gobi 384 Scientific, with the technical characteristics shown in Table 1. This camera has been chosen because of its reduced weight and dimensions, which ease the integration in the system. In addition, the camera is equipped with a $10-\mathrm{mm}$ thermographic lens, which provides a bigger field of view than that of most thermographic cameras. This fact increases the capability of the system to analyse arms in their totality, without the need to move the camera or the arm to complete the study.

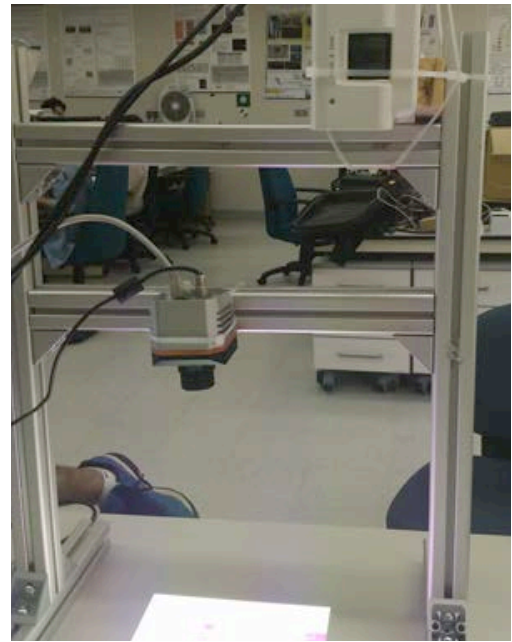

Figure 1. Configuration of the thermographic assistance system

\begin{tabular}{|c|c|}
\hline & Gobi -384 \\
\hline Focal length & $10 \mathrm{~mm}\left(\mathrm{HFOV} 50^{\circ}\right)$ \\
\hline Frame rate & $25 \mathrm{~Hz}$ at 16 bits \\
\hline Array type & Uncooled microbolometer $(\mathrm{a}-\mathrm{Si})$ \\
\hline Spectral band & 8 to $14 \mu \mathrm{m}$ \\
\hline Array size & $384 \times 288$ \\
\hline Pixel pitch & $25 \mu \mathrm{m}$ \\
\hline NETD & $50 \mathrm{~K}$ \\
\hline
\end{tabular}

Table 1. Technical characteristics of thermographic camera integrated in the thermographic assistance system.

However, the system is camera and projector-independent, in such way that any thermographic camera and any projector can be chosen. The conditions for the camera are the availability of an API for the camera to be controlled from the PC as well as to include the vein-detection algorithm for the projection of the resulting image in real-time.
Regarding the projector, any type of device is adequate for its integration in the system, only taking into account that a high resolution of the projector improves the visualisation of the image projected on the arm.

\subsection{System calibration}

The calibration of the system is the most important step for a successful functionality. For this task, a chessboard consisting of circles has been selected, so that the centre of each circle can be detected with subpixel precision (Figure 2). The chessboard chosen is standard from OpenCV, and the position of the centres of the circles is accurately known. The calibration between thermographic camera and the projector is possible because the latter is considered as a pinhole camera for the process.

The chessboard is projected on a cardboard, which is heated by the light projected (Figure 3). Heat transfer is different in the areas with and without circles, in such way that a temperature difference can be detected by the thermographic camera (Figure 2 , right). The cardboard is placed $5 \mathrm{~cm}$ over the support surface, at the same position as the top surface of the arm where the thermographic images will be projected during the study. The distance between the projection plane and the arm-support surface has been established in $5 \mathrm{~cm}$ after the measurement of the arm width of the volunteers.
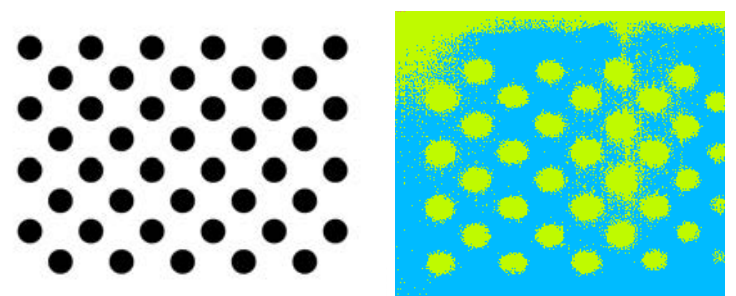

Figure 2. (Left) Pattern of circles. (Right) Thermographic view of the circular pattern.

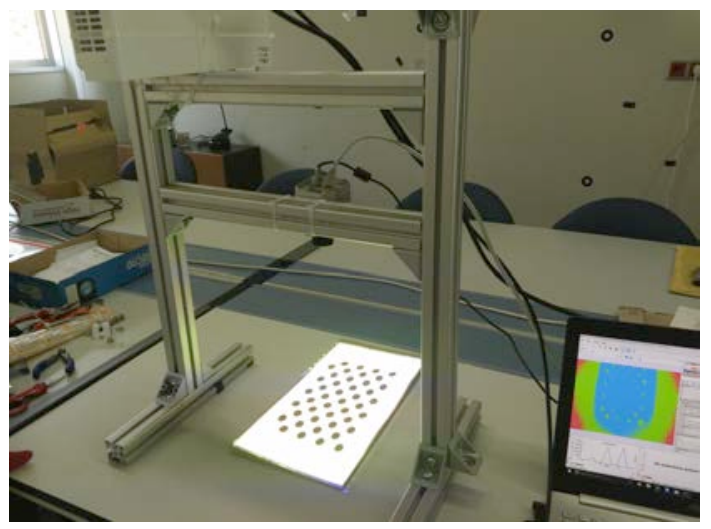

Figure 3. Thermographic-assistance system during calibration (projection of circular pattern and thermographic measurement).

The thermographic image of the chessboard is used for the detection of the centres of the circles, which is performed with subpixel precision. The position of the centres in the image and in the reality is used for the computation of the Fundamental Matrix that relates the position of the two devices (thermographic camera and projector-pinhole camera). The Fundamental Matrix is selected for registration of the devices because, in addition to their relative positions, it includes the intrinsic and extrinsic parameters of the camera, in such way 
that the performance of the geometrical calibration process is avoided (Luong \& Faugeras, 1996).

The Fundamental Matrix is calculated through the Eight-point algorithm, which can be applied because all the points detected in the image are inliers (Hartley, 1997). For the computation of the matrix, the heat transfer and the temperature cycle is not considered for simplification purposes. In addition, the calibration is performed assuming the surface of the arm as a horizontal surface, disregarding the curves of the edges of the arm.

One Fundamental Matrix is calculated for the system, which is possible because the system is a fixed structure. If the positions between projector and thermographic camera are modified, the calibration process should be repeated, computing the corresponding Fundamental Matrix.

\subsection{Thermographic projection}

Once the system is calibrated and the Fundamental matrix between camera and projector is known, the thermographic image can be projected on the arm, with the aim at assisting blood extraction tasks (Figure 4). The projection is performed following Equation 1, which implies the transformation of each pixel of the thermographic image from camera coordinates to projector coordinates.

$$
x=F \times x
$$

where $x$ is the position of each pixel in the projector, $F$ is the Fundamental Matrix and $x^{\prime}$ is the position of the pixel in the thermographic camera.

It should be highlighted that the calibration and projection are established for a horizontal plane $5 \mathrm{~cm}$ over the table or support surface, in order to have an accurate overlap between the image and the arm of the patient. This is an essential task for the success of the process, since the misplacement of the image on the arm would lead to the misguide of the nurse for blood extraction tasks. This parameter can be modified in the configuration of the system, in such way that the system can be adapted to especial cases of wider or thinner arms.

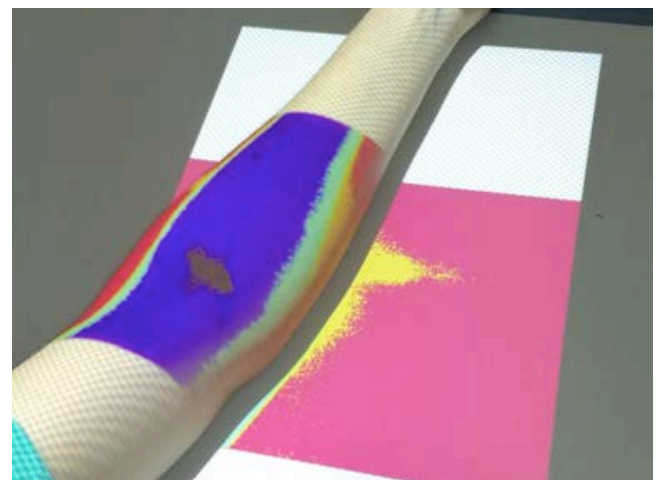

Figure 4. Projection of the thermographic image of the arm for one volunteer (female).

\subsection{Temperature-based cephalic-vein detection}

The algorithm to detect the cephalic-vein in thermographic images is based on the identification of aligned local temperature maximum values, similar to (Cereijo et al, 2014).

The basis of the algorithm is the knowledge of the temperature values corresponding to each pixel in the image. Thus, if the camera provides digital values, its thermal correspondence should be known for the success of the analysis. Once temperature values are available, the images can be analysed as- is, which corresponds to a visualisation in grayscale. A colour palette (RGB) can be applied for viewing purposes, but the use of the converted image in the study increases its complexity and introduces distortion.

Focusing on the algorithm, the first step is the search for aligned local maxima in each image. The condition of alignment is not established since irregularities, curves and misalignments are expected due to the human characteristics, in addition to the configuration of the system and the possibility of placing the arm with different orientation angles regarding the thermographic camera.

In order to eliminate from the detection hot spots not corresponding to veins, a filter based on the Gaussian temperature distribution expected for the veins is applied. Thus, those candidates to veins that do not present a Gaussian but a peak temperature distribution, are dismissed from the process.

No further filters are applied in this case, due to the importance of detecting the vein even partially.

Provided there is no other object in the scene but the arm of the patient, this procedure allows for the detection of the cephalicvein in the arm, regardless the extension of its detectability: from 2-3 pixels to the complete length of the vein (Figure 5).

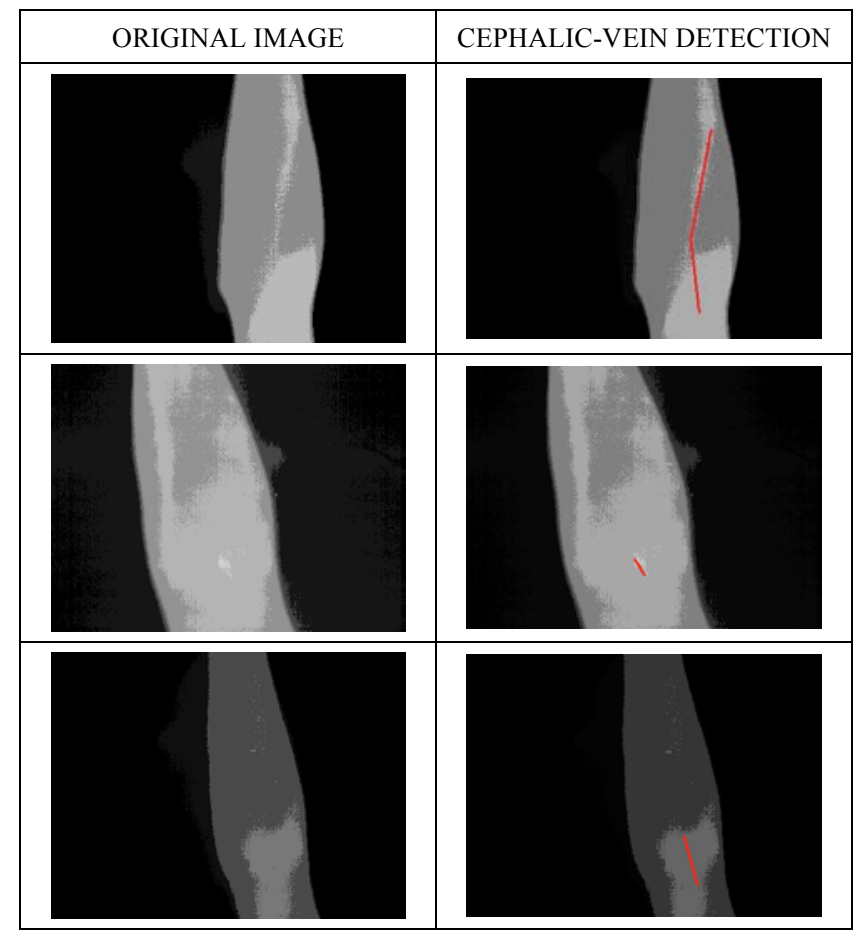

Figure 5. Performance of the algorithm for temperature-based cephalic-vein detection

\section{RESULTS}

The procedure is applied to a set of volunteers, both male and female, with age range between 20 and 50 years old. Various parts of the arm were subjected to the analysis.

\subsection{Cephalic-vein detected in the elbow}

Figure 6 shows example results of the cephalic-vein detected in the area of the elbow, which is the area where the most blood analysis are performed. 


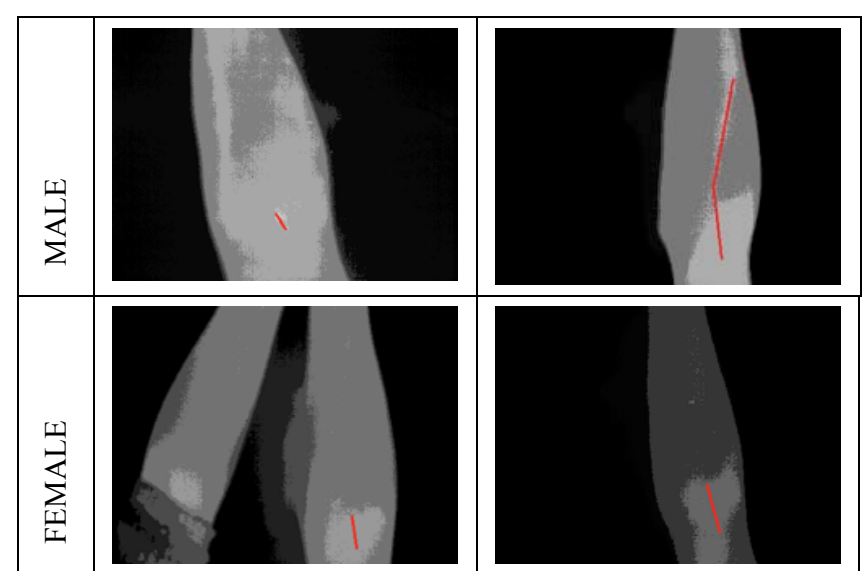

Figure 6. Example of results for the detection of the cephalicvein in male and female volunteers.

The system shows detection results for $100 \%$ of female cases, even when the cephalic-vein is not clearly visible from the thermographic image. In the case of male volunteers, the detection of the cephalic-vein was successful in $70 \%$ of the cases. For the cases where the vein was detected in male volunteers, it was always visible in the thermographic image as a large print at higher temperature.

\subsection{Cephalic-vein detected in the forearm}

The analysis was also performed in the area of the forearm, where blood samples are acquired in those cases when the patient is in a more delicate state and the vein is too weak in the elbow.

The system, as-is, shows detection results only in male volunteers, detecting the vein in the $50 \%$ of the cases (Figure 7). For female volunteers, the system could not detect the cephalicvein down the elbow.

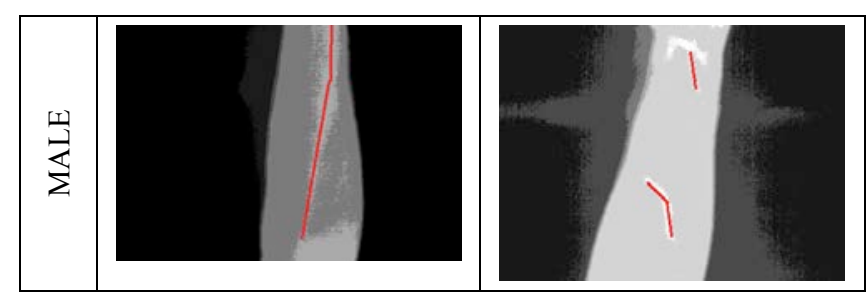

Figure 7. Example of results for the detection of the cephalicvein in the forearm in male volunteers.

The detection of the vein in the forearm is important because the knowledge of its trajectory can determine the location of the vein in the elbow for those cases where the thermographic detection is not possible.

Measurements have also been performed in the arm, with only $5 \%$ successful results. This low rate is attributed to the higher amount of muscle in this area compared to the forearm, which hide the vessels to a deeper position regarding the skin surface.

\section{CONCLUSIONS}

The thermographic-assistance system designed shows successful preliminary results for the detection of the cephalicvein in different patients, regardless the visibility of their vein. This implies an advance for blood tests, since the system can act as an assistant of nurses in the task, especially in those cases where the vein is more weak and difficult to find. The analysis showed that the detection of the vein is not restricted to the area of the elbow, since the application of the algorithm has also shown successful results in the forearm and wrist.

However, authors consider that the percentage of detection can be improved in several ways. Since the study has been performed as passive thermography with no thermal excitation of the arm prior thermographic acquisition, the application of a heat or cold source in the arm can increase the temperature contrast between the skin and the subsurface containing blood vessels.

In addition, further studies should be performed with volunteers in a wider age range, especially including elderly people, which are more usually subjected to blood tests and usually present more difficulties for a successful detection.

Tests should also be applied in more areas where blood extraction is performed, such as the hand-dorsal vein.

Future work will also include the integration of the geometric model of the arm in the process, so that the projection of the thermographic image in the arm presents increased accuracy.

\section{ACKNOWLEDGEMENTS}

Authors would like to thank the Ministry of Economy and Competitiveness, Government of Spain, for the financial support given through human resources grants (IJCI-201524492). Special thanks are given to Eng. Iago Dorado, for its assistance in the technical integration of the system.

\section{REFERENCES}

Alpar, O., Krejcar, O., 2017. Superficial dorsal hand vein estimation. International Conference on Bioinformatics and Biomedical Engineering, pp. 408-418.

Araujo, M., Lima, R., de Souza, R., 2014. Interval symbolic feature extraction for thermography breast cancer detection. Expert Systems with Applications, 41(15), pp. 6728-6737.

Barsom, E., Graafland, M., Schijven, M., 2016. Systematic review on the effectiveness of augmented reality applications in medical training. Surgical Endoscopy, 30(10), pp. 4417-4183.

Borrmann, D., Leutert, F., Schilling, K., Nüchter, A., 2016. Spatial projection of thermal data for visual inspection. In: $14^{\text {th }}$ International Conference on Control, Automation, Robotics and Vision.

Cereijo, J., Lagüela, S., Roca, D., Martínez, J., Lorenzo, H., 2014. Automatic detection of thermal bridges in thermographic building inspections. In: $40^{\text {th }}$ International World Congress on Housing, Funchal, Portugal.

Chakrabory, M., Mukhopadhyay, S., et al, 2016. A new approach of oral cancer detection using bilateral texture features in digital infrared thermal images. In: 2016 IEEE $38^{\text {th }}$ Annual International Conference of the Engineering and Biology Society.

Dayakshini, S., Kamath, S., Rajagopal, K., Prasad, K., 2016. Medical imaging techniques and computer aided diagnostic approaches for the detection of breast cancer with an emphasis on thermography - a review. International Journal of Medical Engineering and Informatics, 8(3), pp. 275-299.

Hartley, R., 1997. In the defense of the eight-point algorithm. IEEE Transactions on Pattern Analysis and Machine Intelligence, 19(6), pp. 580-593. 
Kato, H., Billinghurst, M., 1999. Marker tracking and HMD calibration for a video-based Augmented Reality conferencing system. Proceedings of the $2^{\text {nd }}$ IEEE and ACM International Workshop on Augmented Reality, San Francisco, USA, pp. 8594.

Hildebrandt, C., Raschner, C., Ammer, K., 2010. An overview of recent applications of medical infrared thermography in sports medicine in Austria. Sensors, 10(5), pp. 4700-4715.

Lagüela, S., Díaz-Vilariño, L., Roca, D., 2016. Infrared thermography: Fundamentals and Applications. In: Nondestructive techniques for the evaluation of structures and infrastructure, CRC Press, pp. 113-138.

Luong, Q., Faugeras, O., 1996. The Fundamental Matrix: theory, algorithms, and stability analysis. International Journal of Computer Vision, 17(1), pp. 43-75.

McCafferty, D., 2007. The value of infrared thermography for research on mammals: previous applications and future directions. Mammal Review, 37(3), pp. 207-223.

Thomas, D., 2016, Augmented reality in surgery: the computeraided medicine revolution. International Journal of Surgery, 36, pp. 25-35.

Urakov, A., Urakova, N., Kasatkin, A., Chernova, L., 2014. Physical-chemical aggressiveness of solutions of medicines as factor in the rheology of the blood inside veins and catheters. Journal of Chemistry and Chemical Engineering, 8, pp. 61-65. 